\title{
House Dust Mite Allergy Models-Reliability for Research of Airway Defensive Mechanisms
}

\author{
Tomas Buday, Jana Plevkova \\ Department of Pathophysiology, Jessenius Faculty of Medicine, Comenius University in Bratislava, Martin, \\ Slovakia \\ Email: buday@jfmed.uniba.sk
}

Received 27 May 2014; revised 26 June 2014; accepted 26 July 2014

Copyright (C) 2014 by authors and Scientific Research Publishing Inc.

This work is licensed under the Creative Commons Attribution International License (CC BY). http://creativecommons.org/licenses/by/4.0/

(c) (i) Open Access

\begin{abstract}
Extensive research of respiratory physiology and diseases of the airways and lungs provides better understanding of mechanisms of diseases and it is a source of new data with potential clinical application. The majority of the data in the field of airway defensive reflexes were obtained from guinea pig model, as the most convenient one. Neurophysiology and neuropharmacology of the cough reflex-which is exclusively mediated by the vagus nerve, share close similarities between guinea pigs and humans. The models used to study pathological processes and their influence on airway-defensive reflexes use sensitization with ovalbumin-the protein from chicken egg, which does not mimic allergies within human respiratory system. House dust mites (HDM) (Dermatophagoides pteronyssius and Dermatophagoides farinae) represent frequent human aeroallergens, however the HDM models are used considerably less than ovalbumin models. The primary objective of this review is to focus on already validated models of HDM-induced airway diseases to see, whether some of them are suitable to study mechanisms of peripheral cough plasticity in a condition of HDM-induced pathological processes. The main purpose of future use and validation of HDM model is to produce higher ability to translate the results obtained in animal models to human cough research.
\end{abstract}

\section{Keywords}

House Dust Mite, Allergy, Asthma, Animal Model, Ovalbumin, Cough

\section{Introduction}

Extensive research of respiratory physiology and diseases of the airways and lungs provides better understand-

How to cite this paper: Buday, T. and Plevkova, J. (2014) House Dust Mite Allergy Models-Reliability for Research of Airway Defensive Mechanisms. Open Journal of Molecular and Integrative Physiology, 4, 27-35.

http://dx.doi.org/10.4236/***.2014.***** 
ing of mechanisms of diseases and it is a source of new data with potential clinical application. The key purpose of the basic research is to produce the results with the ability to translate and reproduce the data obtained in HEK (human embryonic kidney) cells, expression systems or animal models to man. The rationale for using animal models in biomedical research is scientific and animal models are likely to remain necessary until science develops alternative models and systems that are equally sound and robust [1]. The data obtained from animal models should be compared and translated to the conditions of human diseases.

Ultimate condition for successful translation is a similarity of the physiology of used animal models and human physiology. However, the substance of studied disease or pathological condition is supposed to be as similar as possible [2].

Cough is the most common symptom of many inflammatory diseases such as asthma and chronic obstructive pulmonary disease (COPD) and as yet, there is no effective treatment (regardless of the cause) to target this symptom. It is also essential to profile, and to establish the efficacy of novel antitussive therapies being developed by the pharmaceutical industry in animal models prior to testing in man. Most pre-clinical studies of neural pathways in the cough reflex and its pharmacological regulation have been conducted in guinea pigs, rats, rabbits, cats and dogs. Although some investigators in the past used the rat and murine models for cough, recent understanding of the airways sensory nerves, regulation of cough limits their use as not relevant models as they lack certain types of nerve endings mediating and modulating cough in humans [3].

One of the most useful and convenient animal models that have been widely used in the last few years and where much information has been gathered regarding the pharmacological modulation of the cough reflex, is the conscious guinea pigs. Extensive work in this field clearly shows that the neurophysiology and neuropharmacology of the guinea pig vagus nerve is very similar to man. A lot of data about the modulation of cough as a defensive mechanisms and nerves involved in their regulatory circuits were obtained from animal models using ovalbumin-induced airway hyperresponsiveness [4]-[7].

Animal models using ovalbumin-induced allergic inflammation and subsequent hyper-sensitivity hyperresponsiveness may have limitations as to translation of the results. Airway hypersensitivity/hyperresponsiveness in humans is mediated by aeroallergens such as pollens or house dust mite (HDM) particles. These allergens probably better mimic the natural airway exposure and therefore may be more suitable models to study airway defense mechanisms on the background of airway inflammation.

The basic concept of this review is to focus on validated models of the house dust mite (HDM)-induced airway hypersensitivity/hyperresponsiveness in guinea pigs to assess, whether they can be successfully used to study the nature and modulation of airway defensive reflexes.

\section{Ovalbumin versus HDM Models}

Nowadays, ovalbumin (OVA) is standard model allergen used in respiratory research in animal models-it induces allergic reaction in animals and even tough OVA-induced allergy reproduces many features of asthmatic lung (e.g. specific IgE levels, Th2-associated eosinophilic inflammation, early (EAR) and late (LAR) asthmatic responses, tissue remodeling of the airway with associated hyperresponsiveness), the clinical significance of ovalbumin as model antigen is dubious.

Ovalbumin models of acute and chronic airway inflammation have been extensively reviewed by Kumar [8] with the list of advantages and disadvantages regarding the clinical application of the data, all together with the description of the limitation of OVA use. OVA, however, is seldom implicated in human asthma, and other groups have used alternative allergens that may have greater clinical relevance, for example house dust mite (HDM) and cockroach extracts [9].

Actually, systemic delivery of OVA (with or without adjuvant-aluminum hydroxide) does not simulate the manner of sensitization to aeroallergens (e.g. pollen, molds or HDM particles) in everyday life. Another problem is that human subjects are basically not allergic to chicken egg protein (ovalbumin) unless it is a food allergy.

One of the alternative models is model of topically applied HDM allergens-a route that, incidentally, is reported to induce tolerance when using OVA. Vast majority of asthmatic patients have increased levels of HDMspecific IgE antibodies and after contact with HDM allergens they manifest with symptoms of asthma (e.g. EAR, LAR and increase in inflammation of airway).

For reasons mentioned, the choice of HDM as model allergen used in sensitization of animal models seems logical-both choice of model antigen and manner of sensitization simulate situation from real life much better than OVA model of airway hypersensitivity. In general, currently used HDM models rely on topical administra- 
tion of HDM into airways (usually intranasally) over course of multiple weeks, which results in airway inflammation accompanied with increased eosinophilia, but there is not enough evidence to show allergic nature of inflammation (HDM-specific IgE antibodies, B- and T-cells). Therefore it is not clear if the inflammation is truly allergic or just a consequence of repeated nasal insults.

In these models, which are based on repeated exposure of model animal to HDM, it is not possible to clearly differentiate between sensitization phase and challenge phase. There would be more reports of occurrence of asthma phenotypes (HDM-specific IgE antibodies, EAR and LAR) if these models have strong allergic component, the same which is observed in OVA- $\mathrm{Al}(\mathrm{OH})_{3}$, which shows specific IgE antibodies and OVA-induced degranulation of mast cells (hyperresponsivity type I). Majority of studies done on HDM models have either assessed specific IgE antibody levels or have reported only weak specific IgE response. This fact is in contrast with clinical experience, where in patients with HDM-induced asthma the levels of specific IgE antibodies is around 100 times higher than in non-allergic individuals. In addition, if specific IgG1 is used as a marker of HDM-specific clonal expansion of B- and T-cells, it seems like this occurs after observation of large airway inflammation (eosinophilia, production of IL-5 and IL-13).

As of now, there is only little knowledge of the role of different cell populations in these models (such as mast cells, dendritic cells, B-cells and Th2-cells) and studying these effectors should be able to elucidate the mechanisms behind repeated HDM sensitization model [10].

\section{House Dust Mites and Their Antigenic Potential}

House dust mite allergens are most important indoor allergens for humans, which induce asthma, rhinitis, dermatitis and other allergic diseases [11]. Mites are part of subclass Acari, where more than 50,000 species are identified to date. For purposes of research, relationship to diseases in humans and as a main perennial indoor allergen, the mites are of particular interest. Most important species, which are the main source of HDM allergens are Dermatophagoides pteronyssius and Dermatophagoides farinae. In addition to these two species, in regions with moderate climate Euroglyphus maynei and in tropical and subtropical areas Blomia tropicalis can be occasionally important. Lepidoglyphus destructor is the source of occupational allergens, which can in some locations contribute to house-dust allergy [12]. The potential to stimulate the immune system to hyperresponsive state of HDM is in mites themselves and in addition, in their faecal pellets as well [13].

HDM induce allergic reaction via allergen specific CD4+ Th2 lymphocytes, however, the role of innate immunity is also critically important. This could be also the reason, why some of the Th2 directed therapeutic approaches have not been successful in clinical trials, because, some other mechanism (outside of the Th2 polarisation) may play an important role.

Immunoblotting studies of HDM allergens in extracts of mites have differed in detail, but in whole there is complexity and the existence of some dominant specificities. Western blotting using sera selected on the basis of anti-mite IgE has revealed 32 different IgE binding sites. Most sera recognized a unique combination of 6 to 10 bands. Out of 32 bands, nine of them were recognized at high frequency. Subsequent study revealed that most sera reacted to 1-7 bands, although some of them did have a broader reactivity spectrum. This pattern is consistent with several similar studies using either $D$. pteronyssius or $D$. farinae. However, the limitations of blotting studies should be recognized - the concentration of allergens vary from extract to extract, some allergens (e.g. Der p2) have their binding site enhanced by denaturation, while others (e.g. Der p1) lose their antigenicity potential and some of the allergens (e.g. Der p 7) show multiple bands [12].

Two groups of HDM allergens have been studied extensively $-25 \mathrm{kDa}$ cysteine protease (group 1) and 14 $\mathrm{kDa}$ epididymal protein (group 2). More than $80 \%$ of humans with HDM allergy mount an IgE response to group 1 and more than $90 \%$ to group 2. However, about $20 \%$ of patients do not have an IgE antibody to either of two group allergens. There are many other HDM allergens containing IgE activity binding sites, although these are present in low and variable concentrations in mite extracts, usually at less than $1 \%$ of the group 1 and 2 allergens - they are therefore called minor allergens [11]. Antigenic potential of HDM is summarized in Figure 1, list of identified allergens from Dermatophagoides pteronyssius and Dermatophagoides farinae is present in Table 1.

\section{HDM versus Airway-An Interaction}

The airway mucosa responds to the presence of HDM particles and the response basically starts with the recognition of the immunogenic epitopes by tissue immune cells, mainly dendritic cells. 


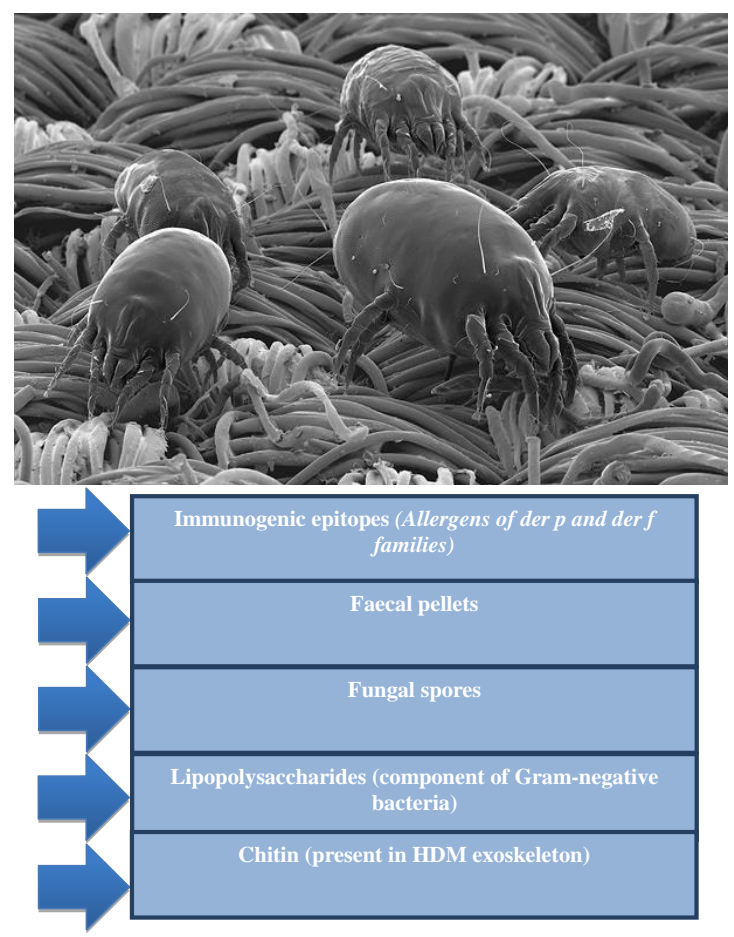

Figure 1. House dust mite allergenicity. Various components of HDM, and their associated faecal pellets and dust, which activate the immune system and initiate an inflammatory response. Modified according to source [13]. Image credit: http://www.blogas.lt/uploads/a/agiesha/204184.jpg

Epithelial cells of bronchi send signals to dendritic cells to induce Th2 immunity to inhaled HDM through release of innate pro-Th2 cytokines (e.g. GM-CSF, TSLP, IL-25 and IL-33) [14]-[16]. Recruitment of eosinophils and IL-4 competent basophils to the lung, which together with activated dendritic cells can traffic to mediastinal lymph nodes is the result of HDM inhalation. Only recently it was shown that FceRI+ dendritic cells are necessity to initiation of Th2 immunity in a model of mucosal sensitization to inhaled HDM allergens and also the role of basophils is limited to amplification of response in asthmatic lung [17].

Protease activity is very common amongst many human allergens, such as fungi, pollen, animal dander or bee venom. HDM and their faecal pellets contain proteolytic enzymes as well—group 1 (Der p 1 and Der $\mathrm{f} 1$ ) are cysteine proteases and groups 3, 6 and 9 (Der p 3, Der p 6, Der p 9, Der f 3, Der f 6 and Der f 9) are serine proteases [18]. Together, they are responsible for 79\% of proteolytic activity of HDM [19]. Der p 1 (Der f 1, respectively) disrupts intercellular tight junctions (proteins occluding and claudin-1 [20]), which allows delivery of antigen to submucosal antigen-presenting cells, whereas the serine proteases activate intracellular degradation of occludin and ZO-1 [21]. This impairment of barrier function of epithelium has not been replicated in murine in vivo model after single dose of HDM [22] as in poorly controlled asthmatic patients [23], which suggests, that this impairment is a result of chronic exposure to HDM, rather than an acute insult.

Moreover, proteases originating from inhaled HDM are able to react with cell surface protease-activated receptors (PARs) in airways, in order to create infiltration of leukocytes and amplification of response to allergens [24]. Activation of these receptors on eosinophils and mast cells leads to their degranulation.

Extracts prepared from mites contain endotoxin. Gram-negative Bartonella sp. are thought to be the source of endotoxin [25]. It can activate both TLR2 and TLR4 directly-these receptors are expressed on epithelium of bronchi and on dendritic cells as well [26] [27]. Moreover, the expression of TLR4 is increased after HDM airway challenge on bronchial epithelium [28]. HDM-induced epithelial TLR4 signaling activates NF- $\mathrm{KB}$ and induces GM-CSF, which in turn results in alveolar macrophage maturation and up-regulation of the co-stimulatory molecules CD40 and CD86. There is a tight correlation between epithelial TLR4 expression and expression of these co-stimulatory molecules on macrophages [28].

TLR activation on epithelial cells also results in trans-activation of epidermal growth factor receptor (EGFR) 
Table 1. Identified allergens of D. pteronyssius and D. farinae [29].

\begin{tabular}{|c|c|c|c|}
\hline \multicolumn{2}{|c|}{ Dermatophagoides pteronyssius } & \multicolumn{2}{|r|}{ Dermatophagoides farinae } \\
\hline Allergen & Biochemical Name & Allergen & Biochemical Name \\
\hline Der $p 1$ & Cysteine protease & Der $\mathrm{f} 1$ & Cysteine protease \\
\hline Der p 2 & NPC2 family & Der f 2 & NPC2 family \\
\hline Der p 3 & Trypsin & Der f 3 & Trypsin \\
\hline Der p 4 & Alpha amylase & & \\
\hline \multicolumn{4}{|l|}{ Der p 5} \\
\hline Der p 6 & Chymotrypsin & Der f 6 & Chymotrypsin \\
\hline Der p 7 & & Der f 7 & \\
\hline Der p 8 & Glutathione s-transferase & & \\
\hline Der p 9 & Collagenolytic serine protease & & \\
\hline Der p 10 & Tropomyosin & Der f 10 & Tropomyosin \\
\hline \multirow[t]{2}{*}{ Der p 11} & Paramyosin & Der f 11 & Paramyosin \\
\hline & & Der f 13 & Fatty acid binding protein \\
\hline Der p 14 & Apolipophorin & Der f 14 & Apolipophorin \\
\hline \multirow[t]{3}{*}{ Der p 15} & Chitinase-like protein & Der f 15 & Chitinase \\
\hline & & Der f 16 & Gelsolin/villin \\
\hline & & Der f 17 & Calcium binding protein \\
\hline Der p 18 & Chitin binding protein & Der f 18 & Chitin binding protein \\
\hline Der p 20 & Arginine kinase & & \\
\hline \multicolumn{4}{|l|}{ Der p 21} \\
\hline & & Der f 22 & \\
\hline \multirow[t]{2}{*}{ Der p 23} & Peritrophin-like protein domain & & \\
\hline & & Der f 24 & Ubiquinol-cytochrome c reductase binding protein homologue \\
\hline
\end{tabular}

[30] which can result in cell migration and production of proteins involved in innate immune response including cytokines and mucins.

Mites carry fungal spores on their surface and also, the fungi are inhabitants of their gut [31]. C-type lectin receptors (dectin-1 and -2) are present of surface of myeloid cells. Their role is to function as classic PRRs by binding to $\beta$-glucans, which are present in fungal and bacterial cell walls, thus creating a link between innate and adaptive responses. Exposure of airway epithelium in vitro to HDM leads to $\beta$-glucan-dependent production of CCL20 [32], which is a chemokine for immature dendritic cells. Extract prepared from dust mites is a specific stimulus for this chemokine and its levels are not affected by cell treatment with either purified Der p 1, chitin, endotoxin or other allergen extracts.

Chitin is the main component of fungal cell wall. It also provides rigidity of exoskeleton of many species (e.g. crustaceans, helminthes, insects and mites). Chitin is fairly potent adjuvant, which regulates both innate and adaptive (Th1, Th2 and Th17) immune responses [33]. It also acts as an element of recognition of tissue infiltration by cells responsible for innate immunity and also induces accumulation of basophils, which express IL-4 in murine model [34]. Exogenous chitin can stimulate macrophages by interacting with different cell surface receptors including TLR2 and dectin-1, thus providing another pathway by which HDM can elicit activation of immune system Allergens Der p 15 and Der p 18 are chitinases. These antigens display high frequency of binding to IgE antibodies in human sera-they are detected in up to 70\% of HDM-allergic patients, which identifies them as HDM allergens of potential high importance [35].

In addition to mite-derived allergens, innate pattern recognition receptors (PRR) derived from microbial compounds, including lipopolysaccharide and $\beta$-glucans are also routinely found in HDM extracts. Moreover, chitin, a glucosamine-based polymer that forms the mite exoskeleton as well as fungal cell walls also stimulates the immune system [13]. 
Mite faecal pellets consist of three to five balls that contain food, debris and proteolytic enzymes. They are bound together by mucus and are covered in a chitinous peritropic membrane and egested. In a lifetime of 10 weeks, a house dust mite will produce approximately 2000 faecal particles (of average diameter 10 to $40 \mu \mathrm{m}$ ) and even larger number of partially digested enzyme-covered dust particles, which can be inhaled and deposited in the conducting airways [13].

\section{HDM Rhinitis Model}

Studies using mice, especially transgenics and knockout strains, have been important for understanding the role of cytokines, adhesion molecules and cell receptors in allergic inflammatory responses [36].

Preclinical research on allergic airway disease is focused predominately on lower airways and asthma-animal models of allergic rhinitis are relatively underdeveloped and understudied by comparison. Until recently, allergic rhinitis models have relied on short-term protocols and therefore present weaknesses of focusing mainly on acute inflammation and less attention is drawn on airway remodelling. However, more effort is being put into developing chronic models to address nasal obstruction, rhinorrhoea and remodelling which define allergic rhinitis in humans [36].

Primary laboratory animals used to describe nasal responses to allergic stimuli are Dunkin-Hartley guinea pigs and, to lesser extent, the Brown Norway rats and BALB/c mice [36].

Nasal obstruction as response to allergic stimulus is characterized by early and late phases of inflammation. An immediate and transient episode of itching and sneezing begins within seconds of exposure and lasts for 5 up to 30 minutes. Late phase is characterized by rhinorrhoea and airway obstruction that can last for hours [36].

The initial irritation and sneeze reflex is promoted by mediators released from mast cells and basophils (histamine, tryptase, cysteinyl leukotrienes, platelet activating factor). Mucus hypersecretion with airway obstruction in secondary phase is accompanied by progression in mucosal swelling, tissue infiltration of eosinophils and neutrophils and the synthesis and release of prostaglandins, interleukins and reactive oxygen species [36].

In allergic guinea pigs, the enumeration of nasal rubbings is subjective but useful measure, especially for testing early phase mechanisms and therapies involving histamine- and leukotriene-dependent pathways. However, the histopathology in both early and late phases in allergic rhinitis hasn't been extensively studied. This contrasts with extensive descriptions of airway remodelling and pathology that drives analogous responses in lower airways (early and late bronchoconstriction) that are well-studied in mice [36].

\section{HDM Asthma Models}

Various models have been developed by increasing the number of allergen exposures over many weeks-socalled chronic allergen challenge models. OVA as well as other allergens such as HDM extract and/or grass pollen have been used. In many of these studies long-term challenge leads to the development of tolerance and progressive decrease in airway hyperresponsiveness and airway eosinophilia. The induction of tolerance may be dependent on the strain of mouse used and the nature and route of allergen administration [37].

Chronic models have been shown to reproduce some of the hallmarks of asthma such as goblet cell metaplasia, epithelial hypertrophy, subepithelial fibrosis and limited smooth muscle hyperplasia. Certain atypical features are observed in these chronic allergen challenge models that are not observed in humans, for example inflammation is not restricted to the conducting airways in the mouse, whereas it is restricted in humans. In addition, there are few mast cells in the airway walls or epithelium of mice [37].

Despite these concerns, development of a standardized chronic model in mice is important as many of the clinical studies were initiated based on outcomes in primary allergen challenge models [37].

\section{Application to the Cough Research}

Cough is an exclusively vagal reflex mediated by the airway sensory neurons with the bodies located in the vagal nodose and jugular ganglia. These fibers are branching in the lower airways mucosa and their complex sensitivity to either mechanical stimulation or acid (nodose $\mathrm{A} \delta$ fibers) and different chemicals (jugular $\mathrm{C}$ fibres) make them perfect sensors for potentially dangerous events (acid aspiration, foreign body aspiration, accumulation of mucus, or inflammatory mediators). All of these stimuli are relevant to provoke coughing [38].

Our team has studied cough reflex and its modulation under many physiological and pathological conditions 
and in the last ten years there has been a growing pool of evidence about such modulation named cough plasticity. The plasticity of the cough neural pathways may occur at the level of airway sensory nerves mediating the cough reflex, at the level of ganglia of the vagus nerve, and also at the level of brainstem circuits regulating neurogenesis of cough [39]. The result of such a modulation is that certain characteristics of cough reflex are modulated either in quantitative or qualitative aspect. The most important and probably clinically most relevant one is up-regulation of the cough reflex, which is usually a mechanism leading to intractable coughing with poor response to cough medication seriously reducing the patients' quality of life (syndrome of chronic cough, chronic hypersensitive cough, and idiopathic chronic cough concepts) [40] [41].

Neuroplasticity changes at different levels are consequences of ongoing inflammation of various aetiologies, including viral, bacterial allergic and neurogenic inflammation. Inflammatory mediators, cytokines, other signal molecules and nerve growth factors are responsible for the changes in sensory channel protein expressions, changes of the nerve fibre phenotypes, branching and growing of existing nerves-thereby increasing the sensitivity of the airways to the nonspecific stimuli [42] [43].

This hypersensitivity has been repeatedly documented in laboratory conditions and clinical settings in animal models and humans suffering from chronic cough. Since these models are in majority ovalbumin-based, there is a circumstantial suggestion that it may not be relevant for human respiratory tract disease. We do believe that validation of the HDM models for future cough research will be more relevant for translational research in this field.

\section{Acknowledgements}

Supported by VEGA 1/0107/2014 and Comenius University Grant No. UK/120/2014.

\section{References}

[1] Chow, P.K.H., Ng, R.T.H. and Ogden, B.E. (2008) Using Animal Models in Biomedical Research: A Primer for the Investigator. World Scientific, Hackensack.

[2] Howells, D.W., Sena, E.S., Porritt, M.J., Rewell, S., et al. (2008) Can Animal Models of Disease Reliably Inform Human Studies? PLoS Medecine, 7, e1000245

[3] Belvisi, M.G. and Bolser, D.C. (2002) Summary: Animal Models for Cough. Pulmonary Pharmacology \& Therapeutics, 15, 249-250. http://dx.doi.org/10.1006/pupt.2002.0349

[4] Mokry, J., Joskova, M., Mokra, D., Christensen, I. and Nosalova, G. (2013) Effects of Selective Inhibition of PDE 4 and $\mathrm{PDE}_{7}$ on Airway Reactivity and Cough in Healthy and Ovalbumin-Sensitized Guinea Pigs. Advances in Experimental Medicine and Biology, 756, 57-64. http://dx.doi.org/10.1007/978-94-007-4549-0_8

[5] Sutovska, M., Adamkov, M., Kocmalova, M., Mesarosova, L., Oravec, M. and Franova, S. (2013) CRAC Ion Channels and Airway Defense Reflexes in Experimental Allergic Inflammation. Advances in Experimental Medicine and Biology, 756, 39-48. http://dx.doi.org/10.1007/978-94-007-4549-0_6

[6] Hori, A., Fujimura, M., Ohkura, N. and Tokuda, A. (2011) Involvement of Nitric Oxide (NO) in Cough Reflex Sensitivity between Non-Sensitized and OVA-Sensitized Guinea Pigs. Cough, 7, 5. http://dx.doi.org/10.1186/1745-9974-7-5

[7] Brozmanova, M., Plevkova, J., Tatar, M. and Kollarik, M. (2008) Cough Reflex Sensitivity Is Increased in the Guinea Pig Model of Allergic Rhinitis. Journal of Physiology and Pharmacology, 59, 153-161.

[8] Kumar, R.K., Herbert, C. and Foster, P.S. (2008) The “Classical” Ovalbumin Challenge Model of Asthma in Mice. Current Drug Targets, 9, 485-494. http://dx.doi.org/10.2174/138945008784533561

[9] Nials, A.T. and Uddin, S. (2008) Mouse Models of Allergic Asthma: Acute and Chronic Allergen Challenge. Disease Models Mechanisms, 1, 213-220 http://dx.doi.org/10.1242/dmm.000323

[10] Birrell, M.A., Van Oosterhout, A.J.M. and Belvisi, M.G. (2010) Do the Current House Dust Mite-Driven Models Really Mimic Allergic Asthma? European Respiratory Journal, 36, 1220-1223 http://dx.doi.org/10.1183/09031936.00069110

[11] An, S., Chen, L., Long, C., Liu, X., Xu, X., Lu, X., Rong, M., Liu, Z. and Lai, R. (2013) Dermatophagoides farinae Allergens Diversity Identification by Proteomics. Molecular \& Cellular Proteomics, 12, 1818-1828. http://dx.doi.org/10.1074/mcp.M112.027136

[12] Thomas, W.R. and Smith, W. (1998) House-Dust-Mite Allergens. Allergy, 53, 821-832. http://dx.doi.org/10.1111/j.1398-9995.1998.tb03987.x

[13] Gregory, L.G. and Lloyd, C.M. (2011) Orchestrating House Dust Mite-Associated Allergy in the Lung. Trends in Im- 
munology, 32, 402-411. http://dx.doi.org/10.1016/j.it.2011.06.006

[14] Barrett, N.A., et al. (2009) Dectin-2 Recognition of House Dust Mite Triggers Cysteinyl Leukotriene Generation by Dendritic Cells. The Journal of Immunology, 182, 1119-1128. http://dx.doi.org/10.4049/jimmunol.182.2.1119

[15] Hammad, H. and Lambrecht, B.N. (2008) Dendritic Cells and Epithelial Cells: Linking Innate and Adaptive Immunity in Asthma. Nature Reviews Immunology, 8, 193-204. http://dx.doi.org/10.1038/nri2275

[16] Lambrecht, B.N. and Hammad, H. (2009) Biology of Lung Dendritic Cells at the Origin of Asthma. Immunity, 31, 412424. http://dx.doi.org/10.1016/j.immuni.2009.08.008

[17] Hammad, H., et al. (2010) Inflammatory Dendritic Cells—Not Basophils—Are Necessary and Sufficient for Induction of Th2 Immunity to Inhaled House Dust Mite Allergen. The Journal of Experimental Medicine, 207, 2097-2111. http://dx.doi.org/10.1084/jem.20101563

[18] Chapman, M.D., et al. (2007) Proteases as Th2 Adjuvants. Current Allergy and Asthma Reports, 7, 363-367. http://dx.doi.org/10.1007/s11882-007-0055-6

[19] Stewart, G.A., et al. (1994) Immunobiology of the Serine Protease Allergens from House Dust Mites. American Journal of Industrial Medicine, 25, 105-107. http://dx.doi.org/10.1002/ajim.4700250128

[20] Wan, H., et al. (1999) Der p 1 Facilitates Transepithelial Allergen Delivery by Disruption of Tight Junctions. Journal of Clinical Investigation, 104, 123-133. http://dx.doi.org/10.1172/JCI5844

[21] Wan, H., et al. (2001) The Transmembrane Protein Occludin of Epithelial Tight Junctions Is a Functional Target for Serine Peptidases from Faecal Pellets of Dermatophagoides Pteronyssinus. Clinical \& Experimental Allergy, 31, 279294. http://dx.doi.org/10.1046/j.1365-2222.2001.00970.x

[22] Turi, G.J., et al. (2011) The Effects of Inhaled House Dust Mite on Airway Barrier Function and Sensitivity to Inhaled Methacholine in Mice. American Journal of Physiology-Lung Cellular and Molecular Physiology, 300, L185-L190. http://dx.doi.org/10.1152/ajplung.00271.2010

[23] Bhure, U.N., et al. (2009) Lung Epithelial Permeability and Inhaled Furosemide: Added Dimensions in Asthmatics. Annals of Nuclear Medicine, 23, 549-557. http://dx.doi.org/10.1007/s12149-009-0275-z

[24] Reed, C.E. and Kita, H. (2004) The Role of Protease Activation of Inflammation in Allergic Respiratory Diseases. Journal of Allergy and Clinical Immunology, 114, 997-1008. http://dx.doi.org/10.1016/j.jaci.2004.07.060

[25] Valerio, C.R., et al. (2005) Bacterial 16S Ribosomal DNA in House Dust Mite Cultures. Journal of Allergy and Clinical Immunology, 116, 1296-1300. http://dx.doi.org/10.1016/j.jaci.2005.09.046

[26] Hammad, H., et al. (2009) House Dust Mite Allergen Induces Asthma via Toll-Like Receptor 4 Triggering of Airway Structural Cells. Nature Medicine, 15, 410-416. http://dx.doi.org/10.1038/nm.1946

[27] Phipps, S., et al. (2009) Toll/IL-1 Signaling Is Critical for House Dust Mite-Specific Helper T Cell Type 2 and Type 17 [Corrected] Responses. American Journal of Respiratory and Critical Care Medicine, 179, 883-893. http://dx.doi.org/10.1164/rccm.200806-974OC

[28] Hongjia, L., et al. (2010) House Dust Mite Regulate the Lung Inflammation of Asthmatic Mice through TLR4 Pathway in Airway Epithelial Cells. Cell Biochemistry and Function, 28, 597-603. http://dx.doi.org/10.1002/cbf.1697

[29] Allergen Nomenclature: IUIS Allergen Nomenclature Sub-Committee. http://www.allergen.org

[30] Koff, J.L., et al. (2008) Multiple TLRs Activate EGFR via a Signaling Cascade to Produce Innate Immune Responses in Airway Epithelium. American Journal of Physiology-Lung Cellular and Molecular Physiology, 294, L1068-L1075. http://dx.doi.org/10.1152/ajplung.00025.2008

[31] Hay, D.B., et al. (1992) How Relevant Are House Dust Mite-Fungal Interactions in Laboratory Culture to the Natural Dust System? Experimental and Applied Acarology, 16, 37-47. http://dx.doi.org/10.1007/BF01201491

[32] Nathan, A.T., et al. (2009) Innate Immune Responses of Airway Epithelium to House Dust Mite Are Mediated through Beta-Glucan-Dependent Pathways. Journal of Allergy and Clinical Immunology, 123, 612-618. http://dx.doi.org/10.1016/j.jaci.2008.12.006

[33] Lee, C.G., et al. (2011) Role of Chitin and Chitinase/Chitinase-Like Proteins in Inflammation, Tissue Remodeling, and Injury. Annual Review of Physiology, 73, 479-501. http://dx.doi.org/10.1146/annurev-physiol-012110-142250

[34] Reese, T.A., et al. (2007) Chitin Induces Accumulation in Tissue of Innate Immune Cells Associated with Allergy. Nature, 447, 92-96. http://dx.doi.org/10.1038/nature05746

[35] O’Neil, S.E., et al. (2006) The Chitinase Allergens Der p 15 and Der p 18 from Dermatophagoides pteronyssinus. Clinical \& Experimental Allergy, 36, 831-839. http://dx.doi.org/10.1111/j.1365-2222.2006.02497.x

[36] Wagner, J.G. and Harkema, J.R. (2012) From Mouse to Man: Translational Value of Animal Models of Allergic Rhinitis. In: Kowalski, M., Ed., Allergic Rhinitis, InTech, 7-11. http://dx.doi.org/10.5772/25352

[37] Shin, Y.S., Takeda, K. and Gelfand, E.W. (2009) Understanding Asthma Using Animal Models. Allergy, Asthma Immu- 
nology Research, 1, 10-18. http://dx.doi.org/10.4168/aair.2009.1.1.10

[38] Canning, B.J. and Chou, Y.L. (2009) Cough Sensors. I. Physiological and Pharmacological Properties of the Afferent Nerves Regulating Cough. Handbook of Experimental Pharmacology, 187, $23-47$. http://dx.doi.org/10.1007/978-3-540-79842-2_2

[39] Mazzone, S.B. and Canning, B.J. (2002) Synergistic Interactions between Airway Afferent Nerve Subtypes Mediating Reflex Bronchospasm in Guinea Pigs. American Journal of Physiology-Regulatory, Integrative and Comparative Physiology, 283, R86-R98.

[40] Morice, A.H. (2013) Chronic Cough Hypersensitivity Syndrome. Cough, 9, 14. http://dx.doi.org/10.1186/1745-9974-9-14

[41] McGarvey, L.P. (2008) Does Idiopathic Cough Exist? Lung, 186, S78-S81. http://dx.doi.org/10.1007/s00408-007-9048-4

[42] Lieu, T. and Undem, B.J. (2011) Neuroplasticity in Vagal Afferent Neurons Involved in Cough. Pulmonary Pharmacology \& Therapeutics, 24, 276-279. http://dx.doi.org/10.1016/j.pupt.2011.02.003

[43] Chen, C.Y., Joad, J.P., Bric, J. and Bonham, A.C. (2009) Central Mechanisms I: Plasticity of Central Pathways. Handbook of Experimental Pharmacology, 187, 187-201. http://dx.doi.org/10.1007/978-3-540-79842-2_9 
Scientific Research Publishing (SCIRP) is one of the largest Open Access journal publishers. It is currently publishing more than 200 open access, online, peer-reviewed journals covering a wide range of academic disciplines. SCIRP serves the worldwide academic communities and contributes to the progress and application of science with its publication.

Other selected journals from SCIRP are listed as below. Submit your manuscript to us via either submit@scirp.org or Online Submission Portal.
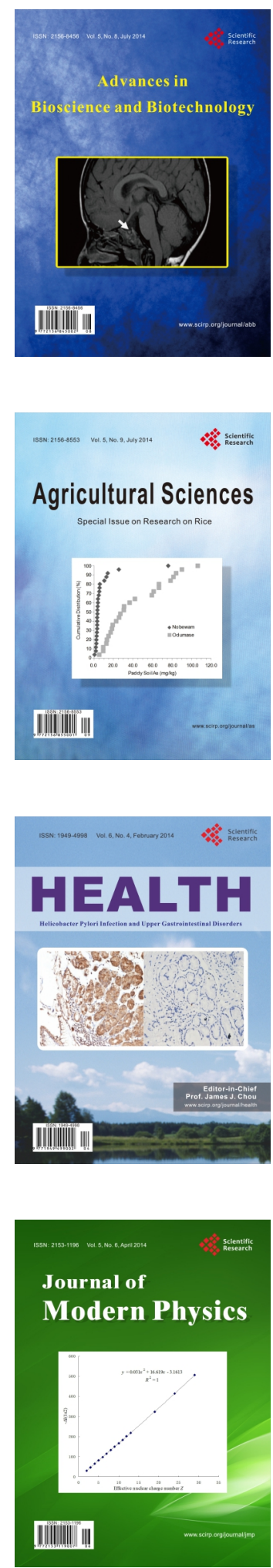
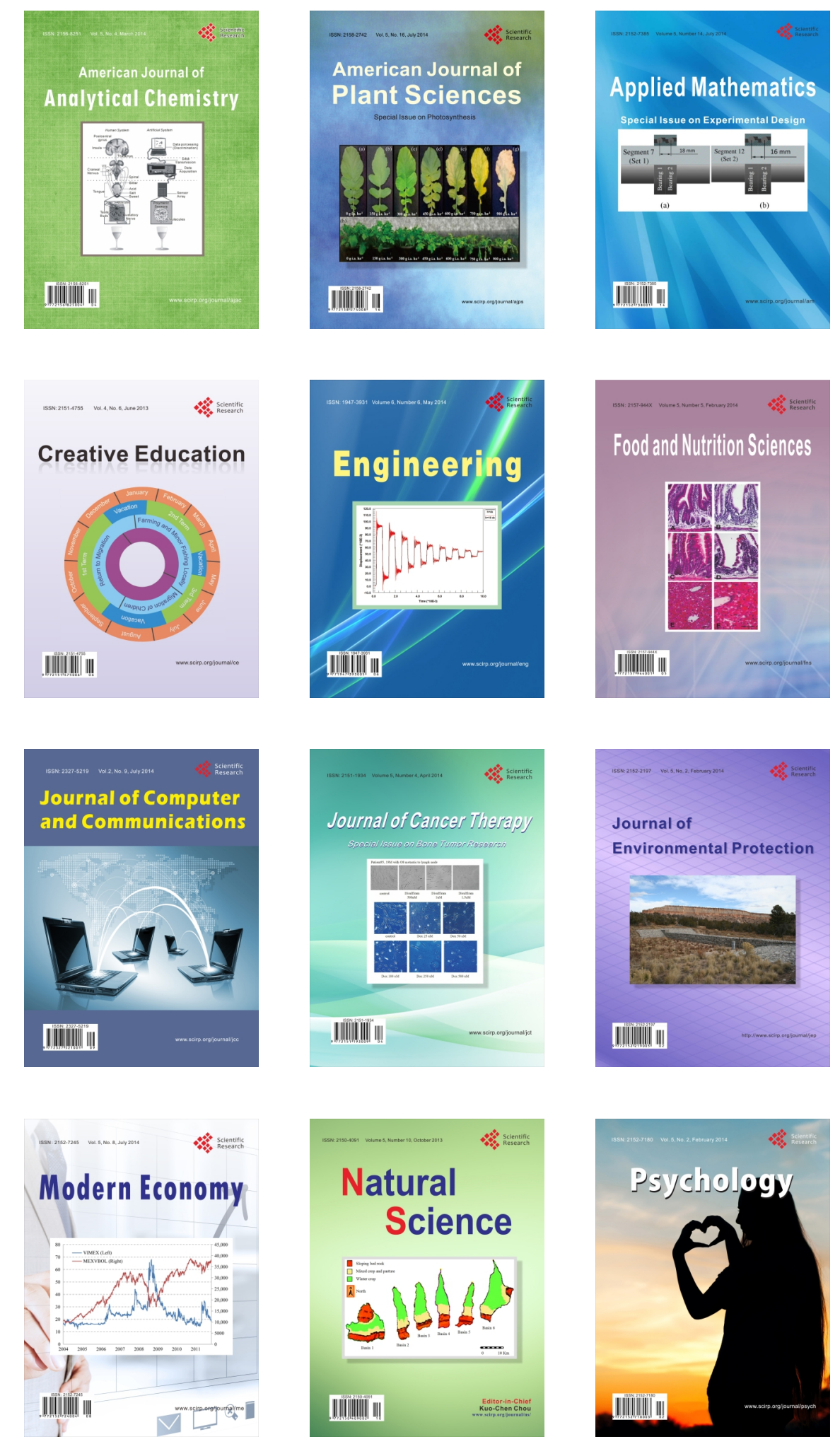